\title{
The functor $T$ and the cohomology of mapping spaces
}

\author{
Jaume Aguadé, Carles Broto, and Laia Saumell
}

\section{Introduction}

In his fundamental work [15] Lannes has introduced a functor $T$ defined in the category $\mathcal{K}$ (resp. $\mathcal{U}$ ) of unstable algebras (resp. modules) over the Steenrod algebra which has many important applications in homotopy theory. This functor is, in some sense, the algebraic analogue of the mapping space functor $\operatorname{Map}(B V,-)$ for an elementary abelian group $V$. More precisely, there is a functor $T=T_{V}: \mathcal{K} \rightarrow \mathcal{K}$ which is adjoint to the functor $-\otimes H^{*}(B V): \mathcal{K} \rightarrow \mathcal{K}$, and which computes, under some hypothesis, $H^{*}\left(\operatorname{Map}\left(B V,-{ }_{p}^{\wedge}\right)\right)$. Here, and throughout this paper, $H^{*}(-)$ denotes $H^{*}\left(-; \mathbb{F}_{p}\right)$ for some fixed prime $p$ and $Y_{p}^{\wedge}$ denotes the $p$-completion of $Y$ in the sense of Bousfield-Kan $([7])$. We also assume that all constructions are done simplicially. For practical purposes, one would like to work on a component of the mapping space containing a particular map $f: B V \rightarrow X$. This is done by considering an algebraic "connected component" of the functor $T$ which we denote $T_{f}$. Hence, we will assume that we have fixed a map $f: B V \rightarrow X$ so that we have a natural transformation

$$
\Gamma: T_{f}\left(H^{*}(X)\right)=T\left(H^{*}(X)\right) \otimes_{\left(T\left(H^{*} X\right)\right)^{0}} \mathbb{F}_{p}^{\{f\}} \rightarrow H^{*}\left(\operatorname{Map}\left(B V, X_{p}^{\wedge}\right)_{f}\right)
$$

which is, under quite general conditions, an isomorphism. Here $\mathbb{F}_{p}\{f\}$ means $\mathbb{F}_{p}$ considered as a module over $T\left(H^{*} X\right)^{0}$ via the restriction to degree zero of the adjoint of the map $f^{*}: H^{*} X \rightarrow H^{*} B V$ and $\operatorname{Map}(Y, Z)_{g}$ denotes the subspace of the mapping space $\operatorname{Map}(Y, Z)$ which contains all maps $g: Y \rightarrow Z$ with $g^{*}=$ $f^{*}: H^{*}(Z) \rightarrow H^{*}(Y)$. (In the cases which we deal with in this paper, this subspace is just the connected component which contains $f$.)

It is clear that some hypothesis on $X$ are needed for $\Gamma$ to be an isomorphism. For instance, if we take $V=0$, the spaces $X$ such that $\Gamma$ is an isomorphism are called $\mathbb{F}_{p}$-good (cf. [7]) and it is known that there are spaces which are not $\mathbb{F}_{p^{-}}$ good (cf. [6]). It is more difficult to find an example in which $X$ is a 1-connected $p$-complete space with $H^{*}(X)$ of finite type and, nevertheless, $T$ does not compute the cohomology of the mapping space. We provide an example of this kind in the last section of this paper.

The authors acknowledge support from DGES grant PB97-0203. 
The relationship between $T_{f} H^{*}(X)$ and $H^{*}\left(\operatorname{Map}\left(B V, X_{p}^{\wedge}\right)_{f}\right)$ was made clear in the work of Dror-Smith ([10]) and Morel ([17], [18]). It turns out that $T_{f} H^{*}(X)$ is isomorphic to the continuous cohomology of the profinite space $\operatorname{Map}(B V, Y)$ where $Y$ is the $p$-completion of the profinite completion of $X$. On the other side, there are some well known conditions on the low dimensional behavior of $T_{f} H^{*}(X)$ which imply that $\Gamma$ is an isomorphism. Lannes $([15])$ proves that $\Gamma$ is an isomorphism if $H^{*}(X)$ and $T_{f} H^{*}(X)$ are of finite type and $T_{f} H^{*}(X)$ vanishes in degree 1 or, more in general, if $T_{f} H^{*}(X)$ is free in degrees $\leq 2$ (see [15], 3.2). The purpose of this paper is to find more general conditions which imply also that $\Gamma$ is an isomorphism.

In [1] it was necessary to compute the cohomology of some mapping spaces in cases in which the functor $T$ does not vanish in degree one and where Lannes' freeness condition does not hold. The problem was solved there by some ad hoc method adapted to the specific value of $T_{f} H^{*}(X)$. The same kind of problem was encountered in [8] and again an ad hoc method was devised to solve it. Once again, along the investigation of the classifying spaces of Kac-Moody groups from a homotopy point of view ([2], see also [3]) it was necessary to compute the cohomology of some mapping spaces in cases when Lannes' condition does not hold.

What we want to do here is to put all these ad hoc methods into a quite general framework in such a way that we can deduce from a single theorem all computations of the cohomology of mapping spaces that we have just mentioned as well as some other computations which could arise. In section 3 we introduce a condition - which looks rather technical but has many direct applicationscalled T-representability or finite T-representability and then Theorem 3, our main theorem, states that finite $T$-representability together with the usual finiteness conditions is a sufficient condition for the homomorphism

$$
\Gamma: T_{f}\left(H^{*}(X)\right) \rightarrow H^{*}\left(\operatorname{Map}\left(B V, X_{p}^{\wedge}\right)_{f}\right)
$$

to be an isomorphism.

This condition of $T$-representability looks much more complex than Lannes' freeness condition. However, we show in sections 4 and 5 of this paper how this condition of $T$-representability can be deduced from the low-dimensional behavior of $T_{f}\left(H^{*}(X)\right)$. This provides us with several concrete examples of cases in which the functor $T$ effectively computes the cohomology of the mapping space. We recover Lannes' criterion, as well as the cases which have been studied in [1], [8] and [2]. Our arguments here heavily depend on group cohomology calculations. The main idea in sections 4 and 5 is to reproduce the low dimensional behavior of $T_{f} H^{*}(X)$ by the cohomology of a finite $p$-group $P$.

Given a $p$-complete space $X$ and a map $f: B V \rightarrow X$, the structure in low dimensions of $T_{f}\left(H^{*}(X)\right)$ is formally captured by an auxiliary algebra $L$ that we define as follows. Let $W_{1}^{*}$ denote the $\mathbb{F}_{p}$-vector space of elements of degree one of $T_{f}\left(H^{*}(X)\right)$. Adjoint to the inclusion it is defined a map of unstable algebras over the Steenrod algebra $U\left(W_{1}^{*}\right) \rightarrow T_{f}\left(H^{*}(X)\right)$, where $U$ is Steenrod's free unstable algebra functor. If $Q_{2}^{*}$ denotes the kernel of this map in degree two, we obtain 
a factorization $U\left(W_{1}^{*}\right) / / U\left(Q_{2}^{*}\right) \rightarrow T_{f}\left(H^{*}(X)\right)$ which is an isomorphism in degree one and a monomorphism in degree two. We define $L=U\left(W_{1}^{*}\right) / / U\left(Q_{2}^{*}\right)$.

To one such algebra $L$, we attach a system of finite $p$-groups $\mathcal{C}(L)$ by the property that their mod $p$ cohomology algebras mimic the behavior of $L$ in low dimensions; more precisely, a finite $p$-group belongs to $\mathcal{C}(L)$ if there is a homomorphism of unstable algebras over the Steenrod algebra $\rho: L \rightarrow H^{*}(P)$ which is an isomorphism in degree one and a monomorphism in degree two. The particular nature of the system $\mathcal{C}(L)$ would imply $T$-representability and even finite $T$-representability. The case in which the system consists of only one finite $p$-group behaves particularly well and is developed independently in section 4 . In cases in which we can deduce finite $T$-representability, we also show that the fundamental group of $\operatorname{Map}\left(B V, X_{p}^{\wedge}\right)_{f}$ is either a finite $p$-group of the system or a pro-finite $p$-group obtained as a limit of a chain in the system.

As said before, the paper ends with an example where $T$ does not compute the cohomology of $\operatorname{Map}\left(B V, X_{p}^{\wedge}\right)_{f}$ for some 1-connected space $X$ of finite type. This example helps in understanding the scope of the main theorem.

We are grateful to F.-X. Dehon for his comments on a previous version of this paper.

\section{Finiteness conditions and the geometric interpretation of $T$}

To simplify the notation, let us say that a connected space $Y$ is of finite $\widehat{\mathbb{Z}}_{p}$-type if $\pi_{1}(Y)$ is a finite $p$-group and $\pi_{i}(Y)$ is a finitely generated $\widehat{\mathbb{Z}}_{p}$-module for all $i>1$. Let us say that $Y$ is of finite $\mathbb{F}_{p}$-type if $H^{i}(Y)$ is a finite dimensional $\mathbb{F}_{p}$-vector space for all $i$. For 1-connected, $p$-complete spaces, both conditions are equivalent (see [1], 5.7). On the other side, if $Y$ is of finite $\widehat{\mathbb{Z}}_{p}$-type then $Y$ is $p$ complete and of finite $\mathbb{F}_{p}$-type. To prove this, consider the universal cover of $Y$, $\widetilde{Y} \rightarrow Y \rightarrow K\left(\pi_{1}(Y), 1\right) . \widetilde{Y}$ is of finite $\mathbb{F}_{p^{-}}$type and $\pi_{1}(Y)$ is a finite $p$-group. Hence, by [7] II.5.2 the fibration is nilpotent in the sense of [7], II.4 and $Y$ is $p$-complete. Then, the Serre spectral sequence shows that $Y$ has also finite $\mathbb{F}_{p}$-type.

We would like to remark that a finitely generated $\widehat{\mathbb{Z}}_{p}$-module is an Ext-pcomplete abelian group in the sense of [7], VI.3. Conversely, an Ext-p-complete abelian group $A$ has a canonical structure of $\widehat{\mathbb{Z}}_{p}$-module given by a natural isomorphism $A \cong \operatorname{Ext}\left(\mathbb{Z}_{p^{\infty}}, A\right)$. This implies that the category of finitely generated $\widehat{\mathbb{Z}}_{p}$-modules is a full subcategory of the category of abelian groups. Moreover, these same ideas can be used to prove that the category of finitely generated $\widehat{\mathbb{Z}}_{p}$-modules is closed under abelian group extensions.

Let us recall also the well known fact that $\lim _{\text {in }}$ an exact functor in the category of towers of finite groups and in the category of towers of finitely generated $\widehat{\mathbb{Z}}_{p}$-modules (Jensen, [14]). Also, a homomorphism of towers of finite groups $\left\{G_{s}\right\} \rightarrow\left\{H_{s}\right\}$ is a pro-monomorphism (pro-epimorphism) if and only if it induces a monomorphism (epimorphism) $\lim _{\longleftarrow} G_{s} \rightarrow \underset{\lim }{\longleftarrow} H_{s}$. 
Finally, we recall the geometric interpretation of the functor $T$ developed by Dror Farjoun-Smith in [10]. Let $X$ be a space such that $H^{*} X$ is of finite type and vanishes in degree one. Then $\pi_{1} X$ is $\mathbb{F}_{p}$-perfect and we get that $X_{p}^{\wedge}$ is simply connected, $p$-complete and $H^{*} X \cong H^{*} X_{p}^{\wedge}$. Also, the homotopy groups of $X_{p}^{\wedge}$ are finitely generated $\widehat{\mathbb{Z}}_{p}$-modules. Let $f: B V \rightarrow X$ be a map and consider $T_{f} H^{*} X$. Let $\left\{P_{s} X_{p}^{\wedge}\right\}$ be the Postnikov tower of $X_{p}^{\wedge}$ and denote $E_{s}=\operatorname{Map}\left(B V, P_{s} X_{p}^{\wedge}\right)_{f_{s}}$ $\left(f_{s}\right.$ is the map induced by $\left.f\right)$. Then Dror Farjoun-Smith prove ([10]) that

$$
T_{f} H^{*} X \cong \lim _{\longrightarrow} H^{*}\left(E_{s}\right),
$$

the isomorphism being induced by the natural map. Hence, the homomorphism

$$
\Gamma: T_{f}\left(H^{*}(X)\right) \rightarrow H^{*}\left(\operatorname{Map}\left(B V, X_{p}^{\wedge}\right)_{f}\right)
$$

is an isomorphism if and only if $H^{*}\left(\operatorname{holim} E_{s}\right) \cong \lim H^{*}\left(E_{s}\right)$ and so the problem that we are investigating in this note is, in a certain sense, a problem about the commutation of homology and inverse limits.

We claim that the spaces $E_{s}$ are of finite $\widehat{\mathbb{Z}}_{p}$-type. We prove this by induction on $s$. Recall that the spaces $E_{s}$ are defined by $E_{s}=\operatorname{Map}\left(B V, P_{s} X_{p}^{\wedge}\right)_{f_{s}}$. Hence, we have principal fibrations $E_{s} \rightarrow E_{s-1}$ whose fibre is a union of components from $\operatorname{Map}\left(B V, K\left(\pi_{s} X_{p}^{\wedge}, s\right)\right)$. Each component is a product of Eilenberg-MacLane spaces $K\left(H^{s-j}\left(B V ; \pi_{s} X_{p}^{\wedge}\right), j\right), 1 \leq j \leq s$. Notice that $X_{p}^{\wedge}$ is simply connected. Hence, if we take into account the remarks on the category of finitely generated $\widehat{\mathbb{Z}}_{p}$-modules that we have made above, the homotopy long exact sequence of these fibrations yields the result by induction.

\section{3. $T$-representability}

Here is our condition on the low dimensional behavior of $T_{f} H^{*} X$ :

Definition 1. We say that $f: B V \rightarrow X$ is T-representable if there is an increasing sequence $\alpha(s)$ and a map of towers

$$
g:\left\{E_{\alpha(s)}\right\} \rightarrow\left\{B_{s}\right\}
$$

such that

1. $B_{s}=K\left(G_{s}, 1\right)$ and $G_{s}$ is a finite p-group for all $s$.

2. $g$ induces a pro-isomorphism in $H_{1}$ and a pro-epimorphism in $\mathrm{H}_{2}$.

3. $H^{*}\left(\lim _{\longleftarrow} G_{s}\right) \cong \lim _{\longrightarrow} H^{*}\left(G_{s}\right)$, induced by the natural map.

If we have a $T$-representation $g:\left\{E_{\alpha(s)}\right\} \rightarrow\left\{B_{s}\right\}$, then we denote $B_{\infty}=$ holim $B_{s}$ and $G_{\infty}=\lim _{\longleftarrow} G_{s}$. Then, since the groups $G_{s}$ are finite, we have $B_{\infty}=$ $K\left(G_{\infty}, 1\right)$.

Here are some straightforward examples of $T$-representability:

1. If $T_{f} H^{*} X$ vanishes in degree one then we get $T$-representability in a trivial way, by taking $B_{s}=*$. 
2. If $T_{f} H^{*} X$ is free in degrees $\leq 2$ (see [15],3.2) we also get $T$-representability in the following way. Let $W$ be the $\mathbb{F}_{p}$-vector space $T_{f}^{1} H^{*} X$. We can take $\left\{B_{s}\right\}$ to be the constant tower $\{K(W, 1)\}$. Then, conditions 1 and 3 are trivially satisfied and 2 is just the condition of being free in degrees $\leq 2$.

In the next sections, we present some other examples of $T$-representability.

In order to prove that $\Gamma$ is an isomorphism we need that the $T$-representation satisfies some finiteness condition. Notice that a $T$-representation $\left\{E_{\alpha(s)}\right\} \rightarrow\left\{B_{s}\right\}$ produces a structure of $H^{*}\left(G_{\infty}\right)$-module on $T_{f} H^{*} X=\lim _{\longrightarrow} H^{*}\left(E_{s}\right)$.

Definition 2. We say that $f: B V \rightarrow X$ is finitely $T$-representable if $T_{f} H^{*} X$ is of finite type and there is a T-representation $\left\{E_{\alpha(s)}\right\} \rightarrow\left\{B_{s}\right\}$ such that either of these conditions holds true:

1. $G_{\infty}$ is a finite p-group.

2. $H^{*}\left(G_{\infty}\right)$ is of finite type and $\operatorname{Tor}_{H^{*}\left(G_{\infty}\right)}^{* *}\left(T_{f} H^{*} X, \mathbb{F}_{p}\right)$ is finite dimensional in each total degree.

In most practical cases, it happens that $T_{f} H^{*} X$ is free over $H^{*}\left(G_{\infty}\right)$ and the above finiteness condition on Tor holds trivially.

We are now ready to state the main result of this paper which says that finite $T$-representability implies that the functor $T$ computes the cohomology of the appropriate mapping space.

Theorem 3. If $X$ is a space of finite $\mathbb{F}_{p}$-type such that $H^{1} X=0$ and $f: B V \rightarrow X$ is finitely $T$-representable, then

$$
\Gamma: T_{f}\left(H^{*}(X)\right) \rightarrow H^{*}\left(\operatorname{Map}\left(B V, X_{p}^{\wedge}\right)_{f}\right)
$$

is an isomorphism. Furthermore, $\operatorname{Map}\left(B V, X_{p}^{\wedge}\right)_{f}$ is p-complete and if the tower of p-groups $\left\{G_{s}\right\}$ provides a T-representation and we write $G_{\infty}=\longleftarrow \lim _{s}$, then we have $\pi_{1} \operatorname{Map}\left(B V, X_{p}^{\wedge}\right)_{f} \cong G_{\infty}$.

Proof. We want to show that $H^{*}\left(\operatorname{holim} E_{s}\right) \cong \lim _{3} H^{*}\left(E_{s}\right)$. We will work in homology. Notice that if we assume that both $T_{f} H^{*} X=\lim _{\longrightarrow} H^{*}\left(E_{s}\right)$ and $H^{*}\left(B_{\infty}\right)$ are of finite type then we have that $H_{*}\left(\lim _{G_{s}}\right) \cong \varliminf_{H_{*}}\left(G_{s}\right)$ and we need to prove that $H_{*}\left(\operatorname{holim} E_{s}\right) \cong \lim _{*} H_{*}\left(E_{s}\right)$.

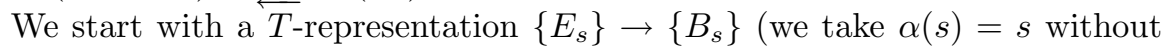
loss of generality) and we denote by $F_{s}$ the homotopy fibre of the map $E_{s} \rightarrow B_{s}$. Then, we split the proof in several steps.

(1) Without loss of generality, we can assume that $F_{s}$ is connected.

To see this, consider $\phi_{s}: \pi_{1} E_{s} \rightarrow \pi_{1} B_{s}$ and define $B_{s}^{\prime}=K\left(\operatorname{Im} \phi_{s}, 1\right)$. We have a map of towers $\left\{E_{s}\right\} \rightarrow\left\{B_{s}^{\prime}\right\}$ and if we could prove that $\left\{B_{s}^{\prime}\right\} \rightarrow\left\{B_{s}\right\}$ is a weak pro-homotopy equivalence ([7], III.3) then it would be clear that $\left\{E_{s}\right\} \rightarrow\left\{B_{s}^{\prime}\right\}$ is also a (finite) $T$-representation with connected fibers.

Recall now that the maximal subgroups of a finite $p$-group are normal and of index $p$. Then, define $H_{s} \triangleleft G_{s}$ to be equal to $G_{s}$ if $\phi_{s}: \pi_{1} E_{s} \rightarrow \pi_{1} B_{s}$ is onto and to be a maximal subgroup of $G_{s}$ containing the image of $\pi_{1} E_{s}$ if $\phi_{s}$ is not onto. We 
want to show that $\left\{H_{s}\right\} \rightarrow\left\{G_{s}\right\}$ is a pro-epimorphism i.e. we need to show that $\lim _{G_{s}} / H_{s}=1$. By hypothesis, $\left\{H_{1} E_{s}\right\} \rightarrow\left\{H_{1} G_{s}\right\}$ is a pro-epimorphism and so

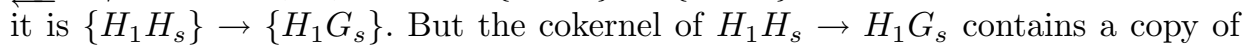
$G_{s} / H_{s}$.

(2) The tower $\left\{H_{1} F_{s}\right\}$ is pro-trivial.

We have for each $s$ a fibration $F_{s} \rightarrow E_{s} \rightarrow B_{s}$ with connected fibre. Notice that The spaces $F_{s}$ are of finite $\widehat{\mathbb{Z}}_{p}$-type and so they are $p$-complete and of finite $\mathbb{F}_{p^{-}}$ type. Hence, to prove that $\left\{H_{1} F_{s}\right\}$ is pro-trivial we can prove that the (ascending)

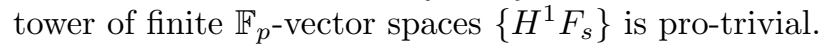

Let us consider the cohomology Serre spectral sequences $E_{*}^{*, *}(s)$ of the fibrations $F_{s} \rightarrow E_{s} \rightarrow B_{s}$. Let $\alpha \in E_{2}^{0,1}(s)=H^{0}\left(B_{s} ; H^{1} F_{s}\right)=\left(H^{1} F_{s}\right)^{G_{s}}$ and let us consider $d \alpha \in H^{2} B_{s}$. Since $\left\{H^{2} B_{s}\right\} \rightarrow\left\{H^{2} E_{s}\right\}$ is a pro-monomorphism, if we take $s$ large enough we can assume $d \alpha=0$. Hence, $\alpha$ represents an element in $H^{1} E_{s}$ not in the image of $H^{1} B_{s}$. Since $\left\{H^{1} B_{s}\right\} \rightarrow\left\{H^{1} E_{s}\right\}$ is a pro-epimorphism, again if we take $s$ large enough we see that $\alpha=0$.

Thus, we have seen that for any $s$ there is $h$ such that

$$
\left(H^{1} F_{s}\right)^{G_{s}} \rightarrow\left(H^{1} F_{s+h}\right)^{G_{s+h}}
$$

is trivial. We finish now with a lemma:

Lemma 4. Let $\left\{V_{i}\right\}$ be an ascending tower of $\mathbb{F}_{p}$-vector spaces. Let $\left\{G_{i}\right\}$ be a descending tower of finite p-groups. Assume that each $V_{i}$ is a $G_{i}$-module and that the obvious coherence condition is satisfied. Then, if the tower of invariants $\left\{V_{i}^{G_{i}}\right\}$ is pro-trivial then the tower $\left\{V_{i}\right\}$ is also pro-trivial.

Proof. Recall that when a finite $p$-group $G$ acts on an $\mathbb{F}_{p}$-vector space $V$, then there is a finite filtration of $V$ by $G$-submodules, $0=V^{(0)} \subset V^{(1)} \subset \cdots \subset V^{(k)}=V$ such that $G$ acts trivially on each quotient $V^{(i)} / V^{(i-1)}$.

In our case, we want to prove that for any $s$ there is $N$ such that $V_{s} \rightarrow V_{s+N}$ is the trivial map. We find $N$ by induction along the filtration of $V_{s}$. Assume we have found $h$ such that all elements of $V_{s}^{(i)}$ die in $V_{s+h}$. By hypothesis, all elements in $\left(V_{s+h}\right)^{G_{s+h}}$ die in some $V_{s+h+k}$. Since $V_{s}^{(i+1)} \rightarrow V_{s+h}$ factors through $V_{s}^{(i+1)} / V_{s}^{(i)}$, it factors also through $\left(V_{s+h}\right)^{G_{s+h}}$ and so $V_{s}^{(i+1)} \rightarrow V_{s+h+k}$ is trivial and the induction goes on.

(3) The tower $\left\{H_{i} F_{s}\right\}$ is pro-constant for any $i$.

This statement is the point where the finiteness assumption is needed. To prove that the tower $\left\{H_{i} F_{s}\right\}$ is pro-constant it is enough to show that $\lim _{i} H_{i} F_{s}$ is finite. This is proven using the Serre spectral sequence or the Eilenberg-Moore spectral sequence, depending on which finiteness condition we assume.

If $\lim _{\longleftarrow} G_{s}$ is a finite $p$-group then we use the (homology) Serre spectral sequence in the following way. The fibrations $F_{s} \rightarrow E_{s} \rightarrow B_{s}$ produce an inverse system of Serre spectral sequences of finite dimensional $\mathbb{F}_{p}$-vector spaces

$$
H_{*}\left(B_{s} ; H_{*} F_{s}\right) \Longrightarrow H_{*} E_{s} .
$$


Since $\lim _{\longleftarrow}$ is exact in this context, we get a spectral sequence

$$
\lim _{\longleftarrow} H_{*}\left(B_{s} ; H_{*} F_{s}\right) \Longrightarrow \lim _{\longleftarrow} H_{*} E_{s} .
$$

By hypothesis, this spectral sequence converges to a graded $\mathbb{F}_{p}$-vector space of finite type. Let us investigate the $E^{2}$-term. First of all, we have an isomorphism (use the spectral sequences of theorem 4.4 in [14])

$$
H_{*}\left(\varliminf_{\longleftarrow} G_{s} ; \lim _{\longleftarrow} H_{*} F_{s}\right) \cong \lim _{\longleftarrow} H_{*}\left(\lim _{\longleftarrow} G_{s} ; H_{*} F_{s}\right) .
$$

Now, $H_{i} F_{s}$ is a $\lim G_{s}$-module via $\lim G_{s} \rightarrow G_{s}$ and $G_{s}$ is a finite $p$-group. Hence,

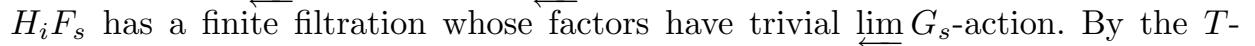
representability hypothesis, $H_{*}\left(\lim _{G_{s}} ; N\right) \cong \lim _{*} H_{*}\left(G_{s} ; N\right)$ if $N$ is a trivial $\mathbb{F}_{p} G_{s^{-}}$ module. Then, by induction along the filtration of $H_{i} F_{s}$ and using the exactness of $\varliminf_{\longleftarrow}$ on finite $\mathbb{F}_{p}$-vector spaces, we get isomorphisms

$$
\lim _{\longleftarrow} H_{*}\left(\varliminf_{\longleftarrow} G_{s} ; H_{*}\left(F_{s}\right)\right) \cong \lim _{r} \lim _{s} H_{*}\left(G_{s} ; H_{*} F_{r}\right) \cong \lim _{s} H_{*}\left(G_{s} ; H_{*} F_{s}\right) .
$$

Hence, we have an spectral sequence starting at $H_{*}\left(\lim _{\leftarrow} G_{s} ; \lim H_{*}\left(F_{s}\right)\right)$ and converging to something of finite type. By hypothesis, $\lim _{\longleftarrow}$ is a finite $p$-group. Then, we can show inductively that $\lim _{*} H_{*}\left(F_{s}\right)$ is of finite type, using the following easy lemma:

Lemma 5. If $G$ is a finite p-group and $M$ is an $\mathbb{F}_{p} G$-module such that $H_{0}(G ; M)$ is finite, then $M$ is finite.

Proof. Let $I$ be the augmentation ideal of $\mathbb{F}_{p} G$. Since $G$ is a finite $p$-group, it is well known that $I^{n}=0$ for some $n$ and so it suffices to prove inductively that $I^{r} M / I^{r+1} M$ is finite. $M / I M=H_{0}(G ; M)$ is finite by hypothesis. The homology exact sequence

$$
H_{1}\left(G ; I^{r} M / I^{r+1} M\right) \rightarrow H_{0}\left(G ; I^{r+1} M\right) \rightarrow H_{0}\left(G ; I^{r} M\right)
$$

yields the inductive step.

If the finiteness condition 2 (involving Tor) is assumed then we use the cohomology Eilenberg-Moore spectral sequence. It converges strongly by [11] because the fundamental group of the base space is $G_{s}$, a finite $p$-group, thus acting nilpotently on the mod $p$ cohomology of the fibre. In the limit, the exactness of lim produces a spectral sequence

$$
\left.\operatorname{Tor}_{\lim }^{*, *} H^{*}\left(G_{s}\right) \stackrel{\left(\lim _{\longrightarrow}\right.}{\longrightarrow} H^{*} E_{s}, \mathbb{F}_{p}\right) \Longrightarrow \quad \longrightarrow \quad \lim _{\longrightarrow} H^{*} F_{s} .
$$

The assumption on Tor implies that $\lim _{\longrightarrow} H^{*} F_{s}$ is of finite type.

(4) holim $F_{s}$ is p-complete, simply connected, and $H_{*}\left(\operatorname{holim} F_{s}\right) \cong \varliminf_{\text {im }} H_{*} F_{s}$.

This is proved as follows. For a space $X$ let us denote by $\left\{R_{s} X\right\}$ the BousfieldKan $\mathbb{F}_{p}$-tower of $X([7])$. Each $R_{s} F_{s}$ is $\mathbb{F}_{p}$-nilpotent, and therefore the coaugmentation of $R_{s}$ induces a weak pro-homotopy equivalence $\left\{R_{s} F_{s}\right\} \rightarrow\left\{R_{s} R_{s} F_{s}\right\}$. Hence, also the map $\left\{R_{s} F_{s}\right\} \rightarrow\left\{R_{s} R_{s} F_{s}\right\}$ obtained by applying the functor $R_{s}$ to $\phi: F_{s} \rightarrow R_{s} F_{s}$ is a weak pro-homotopy equivalence and then, the map of towers 
$\left\{F_{s}\right\} \rightarrow\left\{R_{s} F_{s}\right\}$ induces pro-isomorphisms $\left\{H_{i}\left(F_{s}\right)\right\} \cong\left\{H_{i}\left(R_{s} F_{s}\right)\right\}$ for all $i$ (see [7], III.6.6). Now the tower of fibrations of $\mathbb{F}_{p}$-nilpotent spaces $\left\{R_{s} F_{s}\right\}$ satisfies the hypothesis of lemma 9.3 in [5]. We can conclude that holim $R_{s} F_{s}$ is $p$-complete and simply connected and there are pro-isomorphisms $\left\{H_{i}\left(\operatorname{holim} R_{s} F_{s}\right)\right\} \cong\left\{H_{i}\left(R_{s} F_{s}\right)\right\}$ for all $i$. Since each $F_{s}$ is $p$-complete, $\operatorname{holim} R_{s} F_{s}=\operatorname{holim} F_{s}$. We deduce that there are pro-isomorphisms $\left\{H_{i}\left(\right.\right.$ holim $\left.\left.F_{s}\right)\right\} \cong\left\{H_{i}\left(F_{s}\right)\right\}$ for all $i$ and the claim follows.

(5) Final step.

Consider the inverse system of fibrations $\left\{F_{s} \rightarrow E_{s} \rightarrow B_{s}\right\}$. For simplicity, let us denote $E_{\infty}=\operatorname{holim} E_{s}, F_{\infty}=\operatorname{holim} F_{s}$. Since the homotopy fibre of a map is a particular kind of homotopy limit, we have a fibration $F_{\infty} \rightarrow E_{\infty} \rightarrow B_{\infty}$.

As discussed above, the fibrations $F_{s} \rightarrow E_{s} \rightarrow B_{s}$ produce an inverse system

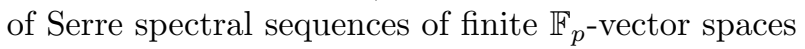

$$
H_{*}\left(B_{s} ; H_{*} F_{s}\right) \quad \Longrightarrow \quad H_{*} E_{s}
$$

and a limit spectral sequence

$$
\lim _{\longleftarrow} H_{*}\left(B_{s} ; H_{*} F_{s}\right) \Longrightarrow \lim _{\longleftarrow} H_{*} E_{s} .
$$

Let us analyze the $E^{2}$-term of this second spectral sequence. He have seen that the tower $\left\{H_{*} F_{\infty}\right\}$ is pro-isomorphic to the tower $\left\{H_{*} F_{s}\right\}$. Notice now that it is easy to show that a homomorphism of towers is a pro-isomorphism if and only if it is a pro-isomorphism of pointed sets. We mean that $\left\{H_{*} F_{\infty}\right\}$ and $\left\{H_{*} F_{s}\right\}$ are also pro-isomorphic as towers of $\mathbb{F}_{p} G_{\infty}$-modules. Hence, the towers $\left\{H_{*}\left(B_{\infty} ; H_{*} F_{\infty}\right)\right\}$ and $\left\{H_{*}\left(B_{\infty} ; H_{*} F_{s}\right)\right\}$ are pro-isomorphic too and this implies that

$$
H_{*}\left(B_{\infty} ; H_{*} F_{\infty}\right) \cong \lim _{*} H_{*}\left(B_{\infty} ; H_{*} F_{s}\right) .
$$

Now, the same argument as in step 3 of the proof shows that

$$
\lim _{\longleftarrow} H_{*}\left(B_{\infty} ; H_{*} F_{s}\right) \cong \lim _{\longleftarrow} H_{*}\left(B_{s} ; H_{*} F_{s}\right)
$$

and the proof of the theorem is complete.

\section{Examples I}

In this section and in the next one we want to show how we can obtain $T$ representability for cohomology algebras, assuming some low dimensional conditions on $T_{f}\left(H^{*}(X)\right)$. As we have already pointed out, if we assume that $T_{f} H^{*}(X)$ is free in degrees $\leq 2$ then we have $T$-representability in a trivial way, by a constant tower $B W$ where $W$ is an $\mathbb{F}_{p}$-vector space, and we want to deal now with a more general situation.

Given two algebras over the Steenrod algebra $A$ and $B$, we will say that a $\mathcal{K}$-map $f: B \rightarrow A$ is an $n$-equivalence if it is bijective in degrees $r<n$ and injective in degree $n$. A sequence of connected algebras over the Steenrod algebra $C \rightarrow B \rightarrow A$ is called an $n$-approximation of $A$ if the composition is trivial in positive degrees and induces an $n$-equivalence $B / / C \rightarrow A$. $T$-representability will be seen to be related to $n$-approximations for small values of $n$. 
If $A$ is a finite type connected unstable algebra over the Steenrod algebra, we can obtain a 2-approximation of $A$ in the following way. Let $W_{1}$ be the $\mathbb{F}_{p^{-}}$ vector space dual to $A^{1}$. The $\mathbb{F}_{p}$-linear map $W_{1}^{*} \rightarrow A$ extends to a $\mathcal{K}$-algebra homomorphism

$$
\varphi: U\left(W_{1}^{*}\right) \rightarrow A
$$

where $U$ is Steenrod's free unstable algebra functor. Let now $Q_{2}$ be the $\mathbb{F}_{p^{-}}$ vector space dual to the kernel of $\varphi$ in degree 2. Then, we have a canonical 2approximation

$$
U\left(Q_{2}^{*}\right) \stackrel{\psi}{\longrightarrow} U\left(W_{1}^{*}\right) \stackrel{\varphi}{\longrightarrow} A .
$$

This 2-approximation produces a central extension of finite $p$-groups in the following way. Let $H_{2}\left(W_{1}\right) \rightarrow Q_{2}$ be dual to the inclusion $Q_{2}^{*} \subset U^{2}\left(W_{1}^{*}\right) \cong H^{2}\left(W_{1}\right)$. This defines an element $\omega \in H^{2}\left(W_{1} ; Q_{2}\right)$ and therefore a central extension of finite p-groups

$$
1 \longrightarrow Q_{2} \stackrel{i}{\longrightarrow} P \stackrel{\pi}{\longrightarrow} W_{1} \longrightarrow 1 \text {. }
$$

Then, one can check by a spectral sequence argument that $\pi$ induces a 2-approximation

$$
U\left(Q_{2}^{*}\right) \stackrel{\psi}{\longrightarrow} U\left(W_{1}^{*}\right) \stackrel{B \pi^{*}}{\longrightarrow} H^{*}(P) .
$$

Notice that this 2-approximation depends only on the structure of the initial algebra $A$ in degrees one and two.

As always throughout this paper, let $X$ be a connected space of finite $\mathbb{F}_{p}$-type with $H^{1}(X)=0$ and let $f: B V \rightarrow X$ be a map. Assume that $T_{f}\left(H^{*}(X)\right)$ is of finite type and let us perform the above construction starting with $A=T_{f}\left(H^{*}(X)\right)$. We obtain a 2-approximation like (2).

Theorem 6. If the 2-approximation (2) associated to $T_{f}\left(H^{*} X\right)$ is a 3-approximation, then $f$ is finitely $T$-representable.

Hence the question of finite $T$-representability is essentially reduced to one about group cohomology. Notice also that if $T_{f}\left(H^{*}(X)\right)$ is free in degrees $\leq 2$ then this theorem applies trivially $\left(Q_{2}^{*}=0\right)$ and so this theorem generalizes Lannes' criterion.

Proof of Theorem 6. The idea here is to prove that the constant tower $\{B P\}$ can be used to prove $T$-representability. Let $\left\{E_{s}\right\}$ be the tower of fibrations with $E_{s}=$ $\operatorname{Map}\left(B V, P_{s} X_{p}^{\wedge}\right)_{f_{s}}$ as in section 2. It provides in $\bmod p$ cohomology a direct system $\left\{H^{*}\left(E_{s}\right)\right\}$ with limit $T_{f}\left(H^{*}(X)\right)$. On the other side, we have the canonical 2approximation

$$
U\left(Q_{2}^{*}\right) \stackrel{\psi}{\longrightarrow} U\left(W_{1}^{*}\right) \stackrel{\varphi}{\longrightarrow} T_{f}\left(H^{*}(X)\right) .
$$


Then, for some large index $s$, there is a factorisation

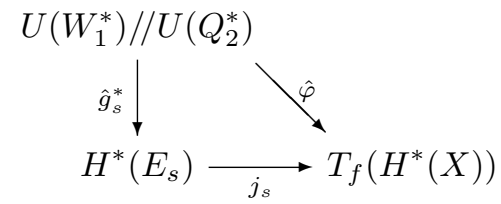

where $g_{s}^{*}: U\left(W_{1}^{*}\right) \rightarrow H^{*}\left(E_{s}\right)$ is induced by a map $g_{s}: E_{s} \rightarrow B W_{1}$ which should lift to $B P$. Hence, we have a diagram

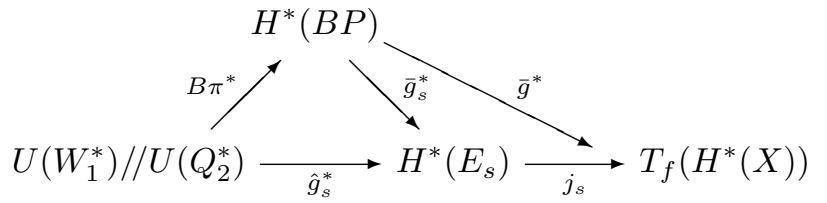

where we defined $\bar{g}^{*}=j_{s} \circ \bar{g}_{s}^{*}$. $B \pi^{*}$ is a 3 -equivalence by hypothesis and therefore $\bar{g}^{*}: H^{*}(B P) \rightarrow T_{f}\left(H^{*}(X)\right)$ is a 2-equivalence. It follows that the obvious map of towers $\left\{E_{s}\right\} \rightarrow\{B P\}$ induces a pro-isomorphism in $H_{1}$ and a pro-epimorphism in $H_{2}$ and so $f: B V \rightarrow X$ is $T$-representable.

The question is now to decide when the sequence

$$
U\left(Q_{2}^{*}\right) \stackrel{\psi}{\longrightarrow} U\left(W_{1}^{*}\right) \stackrel{\pi^{*}}{\longrightarrow} H^{*}(P)
$$

associated to a group extension $Q_{2} \longmapsto P \rightarrow W_{1}$ is a 3 -approximation. This question can be investigated by means of the Serre spectral sequence of the extension. The first differential $d_{2}: E_{2}^{0,1} \rightarrow E_{2}^{2,0}$ is identified with the map $\psi$ in (3) in degree 2 . The next differential $d_{3}: E_{3}^{0,2} \rightarrow E_{3}^{3,0}$ is the transgression

$$
\tau: \beta Q_{2}^{*} \longrightarrow U^{3}\left(W_{1}^{*}\right) / W_{1}^{*} \cdot Q_{2}^{*}
$$

that satisfies $\tau(\beta q)=\beta(\psi(q))$ modulo $\operatorname{Im} \psi$.

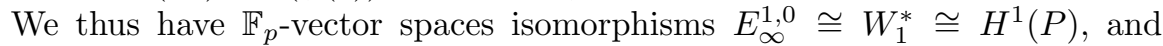
$E_{\infty}^{2,0} \cong U^{2}\left(W_{1}^{*}\right) / Q_{2}^{*} \subset H^{2}(P)$, with complement isomorphic to $E_{\infty}^{1,1} \oplus E_{\infty}^{0,2} ;$ and furthermore, there are exact sequences

$$
\begin{gathered}
0 \longrightarrow E_{\infty}^{1,1} \longrightarrow W_{1}^{*} \otimes Q_{2}^{*} \stackrel{m}{\longrightarrow} U^{3}\left(W_{1}^{*}\right) \longrightarrow U^{3}\left(W_{1}^{*}\right) / W_{1}^{*} \cdot Q_{2}^{*} \longrightarrow 0 \\
0 \longrightarrow E_{\infty}^{0,2} \longrightarrow \beta Q_{2}^{*} \stackrel{\tau}{\longrightarrow} U^{3}\left(W_{1}^{*}\right) / W_{1}^{*} \cdot Q_{2}^{*} \longrightarrow E_{\infty}^{3,0} \subset H^{3}(P),
\end{gathered}
$$

where $m$ is given by multiplication in $U\left(W_{1}^{*}\right): m(\omega \otimes q)=\omega \cdot \psi(q)$.

It follows that the necessary and sufficient condition for (3) to be a 3 approximation is that $m$ and $\tau$ are injective. If $p=2$, then $m$ is always injective because $U\left(W_{1}^{*}\right)$ does not contain zero divisors but for an odd $p$ this is not necessarily so. We have thus proved

Proposition 7. (3) is a 3-equivalence if and only if either $p=2$ and the transgression map $\tau: S q^{1} Q_{2}^{*} \rightarrow U^{3}\left(W_{1}^{*}\right) / W_{1}^{*} \cdot Q_{2}^{*}$ is injective; or $p$ is odd and both the transgression $\tau: \beta Q_{2}^{*} \rightarrow U^{3}\left(W_{1}^{*}\right) / W_{1}^{*} \cdot Q_{2}^{*}$ and $m: Q_{2}^{*} \otimes W_{1}^{*} \rightarrow U^{3}\left(W_{1}^{*}\right)$ are injective. 
We will now look more closely at the particular case in which $Q_{2}^{*}$ is 1dimensional generated by a quadratic form $q \in H^{2}\left(W_{1}\right)$. In this case the extension $\mathbb{Z} / p \longmapsto P \rightarrow W_{1}$ is an extraspecial group and the cohomology of these groups has been largely studied. The mod 2 cohomology of extraspecial 2-groups was described by Quillen ([19]). At odd primes our knowledge is not complete. Diethelm ([9]) and Leary ([16]) computed the mod p cohomology of extraspecial groups or order $p^{3}$. A general reference is a beautiful paper by Benson and Carlson [4].

For $p=2$, if $n$ is the dimension of $W_{1}^{*}$ and $h$ is the codimension of a maximal isotropic subspace of $q$ in $W_{1}^{*}$, we have

$$
H^{*}\left(P ; \mathbb{F}_{2}\right) \cong P\left[x_{1}, x_{2}, \ldots, x_{n}\right] /\left(q_{o}, q_{1}, \ldots, q_{h-1}\right) \otimes P[\zeta]
$$

where $x_{i}$ are all of degree $1, q_{0}=q$ has degree $2, q_{i+1}=S q^{2^{i}} q_{i}$, and $\operatorname{deg} \zeta=2^{h}$.

Corollary 8. Assume that $p=2$ and $T_{f}\left(H^{*}(X)\right)$ has a 2-approximation

$$
U\left(Q_{2}^{*}\right) \stackrel{\psi}{\longrightarrow} U\left(W_{1}^{*}\right) \stackrel{\varphi}{\longrightarrow} T_{f}\left(H^{*}(X)\right)
$$

where $Q_{2}^{*}$ is generated by a single quadratic form $q \in U^{2}\left(W_{1}^{*}\right)$ with the property that a maximal isotropic subspace of $q$ has codimension $h \geq 2$. Then $f: B V \rightarrow X$ is finitely T-representable.

Example 9. If $p=2$ and $T_{f}\left(H^{*}(X)\right)$ has two generators $x, y$ in degree one which satisfy a single relation $q$ in degree 2 , there are three possible cases: $q=x^{2}$, $q=x y$ or $q=x^{2}+x y+y^{2}$. In the first two cases, the codimension of a maximal isotropic subspace is 1 and Corollary 8 does not apply. Here the group $P$ is either $\mathbb{Z} / 4 \mathbb{Z} \times \mathbb{Z} / 2 \mathbb{Z}$ or the dihedral group of 8 elements. Those cases will be considered in next section. In the case in which $q=x^{2}+x y+y^{2}, P$ is the quaternion group of order $8, h=2$ and Corollary 8 applies.

If $p$ is odd, $q \in H^{2}\left(W_{1}\right)$ is not really a quadratic form. We have

$$
H^{2}\left(W_{1} ; \mathbb{F}_{p}\right) \cong \Lambda^{2}\left(W_{1}^{*}\right) \otimes \beta W_{1}^{*},
$$

thus $q$ can be written as $q=s+\lambda$, where $s \in \Lambda^{2}\left(E_{1}^{*}\right)$ is a symplectic form and $\lambda \in \beta W_{1}^{*}$ a linear form.

Corollary 10. Assume that $p$ is odd and $T_{f}\left(H^{*}(X)\right)$ has a 2-approximation

$$
U\left(Q_{2}^{*}\right) \stackrel{\psi}{\longrightarrow} U\left(W_{1}^{*}\right) \stackrel{\varphi}{\longrightarrow} T_{f}\left(H^{*}(X)\right)
$$

where $Q_{2}^{*}$ is generated by a single form $q=s+\lambda \in U^{2}\left(W_{1}^{*}\right)$. Then, if both $s$ and $\lambda$ are non-trivial, $f: B V \rightarrow X$ is finitely T-representable.

Proof. Let $\mathbb{Z} / p \longmapsto P \rightarrow W_{1}$ be the extension classified by $q=s+\lambda$. If we write $H^{*}(\mathbb{Z} / p) \cong P[z] \otimes \Lambda(t)$, the differential $d_{2}$ in the Serre spectral sequence of the extension hits que extension class $d_{2}(t)=q$. If $\lambda \neq 0$, then $q$ is not a zero divisor and $E_{3}^{*, 1}=0$. The next differential is $d_{3}(z)=\beta(q)=\beta(s)$, and if $s \neq 0$, then it is not divisible by $\lambda$. Hence $d_{3}(z) \neq 0$ in $E_{3}^{*, *}$ and $z$ does not survive to $E_{\infty}^{*, *}$. It follows that

$$
U\left(E_{1}^{*}\right) / / U\left(Q_{2}^{*}\right) \longrightarrow H^{*}\left(P ; \mathbb{F}_{p}\right)
$$


is a 3 -equivalence.

Example 11. If $p$ is odd and $T_{f}\left(H^{*}(X)\right)$ has two 1-dimensional generators $y_{1}, y_{2}$, that satisfy a single relation $q \in\left\langle y_{1} y_{2}, x_{1}, x_{2}\right\rangle$, where we write $x_{i}=\beta y_{i}$, then there are three possibilities.

1. If $q=x_{1}$ then $P \cong \mathbb{Z} / p^{2} \times \mathbb{Z} / p$ and Corollary 10 does not apply.

2. If $q=y_{1} y_{2}$ then $P$ is the non-abelian group of order $p^{3}$ and exponent $p$. Its mod p cohomology is described in [16]. Again Corollary 10 does not apply.

3. If $q=y_{1} y_{2}+x_{1}$ then $P$ is now a non-abelian split metacyclic group of order $p^{3}$. The cohomology ring was computed in [9]. Now, Corollary 10 applies.

\section{Examples II: Systems of p-groups with fixed low dimensional cohomology}

This section is devoted to a further investigation of cases in which Theorem 6 does not apply. That is, cases in which the 2-approximation of $T_{f}\left(H^{*}(X)\right)$ determines an extension $Q_{2} \rightarrow P \rightarrow W_{1}$ for which $U\left(W_{1}^{*}\right) / / U\left(Q_{2}^{*}\right) \rightarrow H^{*}\left(P ; \mathbb{F}_{p}\right)$ is not a 3-equivalence (cf. Example 9 for $q=x^{2}, x y$ ).

Fix the algebra $L=U\left(W_{1}^{*}\right) / / U\left(Q_{2}^{*}\right)$. As a first step we attach to $L$ a system of finite $p$-groups, or, more precisely, a system of isomorphism classes of finite p-groups:

$$
\mathcal{C}(L)=\left\{P \text { finite } p \text {-group } \mid \text { there is a 2-equivalence } \rho: L \rightarrow H^{*}(P)\right\}
$$

where we just write $P$ for the isomorphism class that it represents. $\mathcal{C}(L)$ is a poset with the relation $P \lessdot P^{\prime}$ if there is an epimorphism $\pi: P^{\prime} \rightarrow P$. It is useful to observe that this epimorphism induces a commutative diagram in cohomology

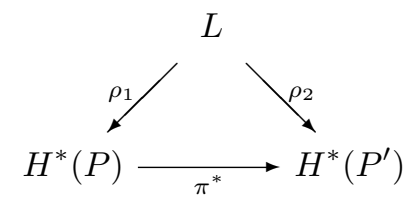

where $\rho_{1}$ and $\rho_{2}$ are 2-equivalences. In fact, if $K$ is the kernel of $\pi$, we have an exact sequence $0 \rightarrow H^{1}(P) \rightarrow H^{1}\left(P^{\prime}\right) \rightarrow H^{1}(K)^{P}$, so that $\pi^{*}$ is a monomorphism in degree 1 , but $H^{1}(P)$ and $H^{1}\left(P^{\prime}\right)$ have the same finite dimension. This shows the commutativity of the diagram in degree 1 . In degree 2 the commutativity of the diagram follows because $L$ is generated by degree one elements.

Now we state some properties of the system $\mathcal{C}(L)$.

(A) If $P \in \mathcal{C}(L)$ and $1 \rightarrow V \rightarrow P^{\prime} \rightarrow P \rightarrow 1$ is a central extension, with $V$ elementary abelian $p$-group, determined by an inclusion $V^{*} \subset H^{2}(P)$, then, $P^{\prime} \in \mathcal{C}(L)$ if and only if $V^{*} \cap \rho\left(L^{2}\right)=\{0\}$. 
(B) A relation $P \lessdot P^{\prime}$ can be refined to a chain $P=P_{1} \lessdot P_{2} \lessdot P_{3} \lessdot \cdots \lessdot P_{r}=P^{\prime}$ in $\mathcal{C}(L)$ such that, for each $k$ there is a central extension $1 \rightarrow V_{k} \rightarrow P_{k} \rightarrow$ $P_{k-1} \rightarrow 1$, with $V_{k}$ elementary abelian $p$-group.

(C) There is an initial element. Namely, the extension given by the 2-approximation $U\left(Q_{2}^{*}\right) \rightarrow U\left(W_{1}^{*}\right) \rightarrow L$.

(D) An element $P$ in $\mathcal{C}(L)$ is maximal if and only if the 2-equivalence $\rho: L \rightarrow$ $H^{*}(P)$ is actually an isomorphism in degree 2 .

We will say that a system $\mathcal{C}(L)$ is of finite type if for any infinite chain

$$
P_{\lambda_{1}} \lessdot P_{\lambda_{2}} \lessdot \cdots \lessdot P_{\lambda_{s}} \lessdot \cdots
$$

the mod $p$ cohomology of the $p$ pro-finite group $P_{\lambda_{\infty}}=\lim _{\longleftarrow} P_{\lambda_{s}}$ is of finite type and $H^{*}\left(P_{\lambda_{\infty}}\right) \cong \lim ^{*} H^{*}\left(P_{\lambda_{s}}\right)$.

Before providing some examples of system of $p$-groups we show how to prove the above properties. For (A), we use the Serre spectral sequence for the extension $1 \rightarrow V \rightarrow P^{\prime} \rightarrow P \rightarrow 1$. In low dimensions it provides an exact sequence

$$
0 \rightarrow H^{1}(P) \rightarrow H^{1}\left(P^{\prime}\right) \rightarrow H^{1}(V) \stackrel{d_{2}}{\longrightarrow} H^{2}(P) \rightarrow H^{2}\left(P^{\prime}\right)
$$

But the differential $d_{2}$ is given by the extension class, so, in our case it is identified with the inclusion $V^{*} \subset H^{2}(P)$ and the exact sequence breaks to an isomorphism $H^{1}(P) \cong H^{1}\left(P^{\prime}\right)$ and a monomorphism $H^{2}(P) / V^{*} \longmapsto H^{2}\left(P^{\prime}\right)$. The composition $L \stackrel{\rho}{\rightarrow} H^{*}(P) \rightarrow H^{*}\left(P^{\prime}\right)$ in degree 2 factors through this monomorphism and this proves the statement.

For (B) we start with an epimorphism $P^{\prime} \rightarrow P$. If it is an isomorphism we are done, otherwise, let $K$ be the kernel. Using the action of $P^{\prime}$ on $K$ by conjugation we can find an elementary abelian subgroup of $K, V$, which is central in $P^{\prime}$, and then we can form the factorization $P^{\prime} \rightarrow P^{\prime} / V \rightarrow P$, and repeat the same argument inductively until we obtain a sequence of epimorphisms $P^{\prime}=P_{r} \rightarrow$ $P_{r-1} \rightarrow \cdots \rightarrow P_{2} \rightarrow P_{1}=P$ where each step is a central extension. We can compose the restrictions with the 2-equivalence $\rho: L \rightarrow H^{*}(P)$ and get a diagram

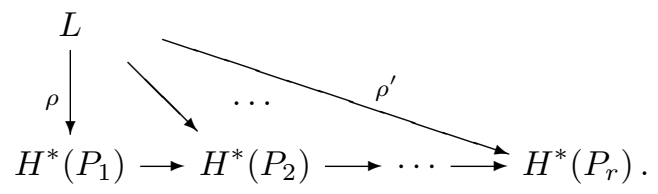

In degree one $\rho$ and $\rho^{\prime}$ are isomorphisms, while the exact sequence (4) applies to each step $V_{k} \longmapsto P_{k} \rightarrow P_{k-1}$ and then the morphisms in the bottom row are all monomorphisms, hence isomorphisms. In degree two (4) now implies that $H^{2}\left(P_{k-1}\right) \rightarrow H^{2}\left(P_{k}\right)$ factors as $H^{2}\left(P_{k-1}\right) \rightarrow H^{2}\left(P_{k-1}\right) / V_{k}^{*} \longmapsto H^{2}\left(P_{k}\right)$ and since both $\rho$ and $\rho^{\prime}$ are injective in degree two, it follows that at each step $V_{k}^{*} \cap \rho_{k}\left(L^{2}\right)=\{0\}$ and then property (A) applies and $P_{k} \in \mathcal{C}(L)$.

We will now show that the central extension $Q_{2} \rightarrow P \rightarrow W_{1}$ determined by $L=U\left(W_{1}^{*}\right) / / U\left(Q_{2}^{*}\right)$ is an initial object in $\mathcal{C}(L)$, thus proving property (C). Assume that $P^{\prime} \in \mathcal{C}(L)$. The 2-equivalence $\rho: L \rightarrow H^{*}\left(P^{\prime}\right)$ gives $W_{1}^{*} \cong H^{1}\left(P^{\prime}\right)$, and then it gives an epimorphism $P^{\prime} \rightarrow W_{1} \cong H_{1}\left(P^{\prime}\right)$. Let $K$ be the kernel. 
The Serre spectral sequence for the extension $1 \rightarrow K \rightarrow P^{\prime} \rightarrow W_{1} \rightarrow 1$ gives an exact sequence $0 \rightarrow H^{1}(K)^{W_{1}} \stackrel{d_{2}}{\longrightarrow} H^{2}\left(W_{1}\right) \rightarrow H^{2}\left(P^{\prime}\right)$. The last homomorphism factors through $L$ in degree $2 H^{2}\left(W_{1}\right) \rightarrow L^{2} \mapsto H^{2}\left(P^{\prime}\right)$ and so, therefore, $Q_{2}^{*} \cong$ $H^{1}(K)^{W_{1}} \subset H^{1}(K)$. The dual of this inclusion provides an epimorphism $K \rightarrow Q_{2}$ and a map of extensions

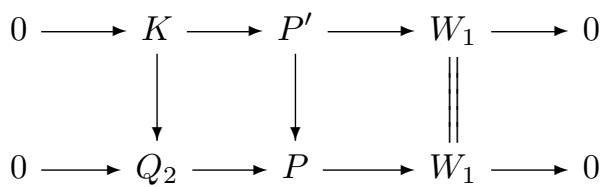

where the middle vertical homomorphism will also be an epimorphism, thus proving $P \lessdot P^{\prime}$.

Finally we prove (D). Assume first that $\rho\left(L^{2}\right) \cong H^{2}(P)$ and that $P^{\prime}$ is another group in $\mathcal{C}(L)$ with $P \lessdot P^{\prime}$. According to (B) there is another group $P^{\prime \prime} \in \mathcal{C}(L)$ and a central extension $V \rightarrow P^{\prime \prime} \rightarrow P$. This extension is classified by an inclusion $V^{*} \subset H^{2}(P)$, that according to (A) satisfies $V^{*} \cap \rho\left(L^{2}\right)=0$. But this can only be true if $V=0$, that is $P^{\prime \prime} \cong P$ and then, also, $P^{\prime} \cong P$.

Conversely, if $\rho\left(L^{2}\right) \nsucceq H^{2}(P)$ there is a non trivial complement $V^{*}$ of $\rho\left(L^{2}\right)$ in $H^{2}(P)$, defining a central extension $V \rightarrow P^{\prime} \rightarrow P$ with $P^{\prime} \in \mathcal{C}(L)$, by $(\mathrm{A})$, so that $P \lessdot P^{\prime}$ with $P \nsubseteq P^{\prime}$ and $P$ is not maximal in $\mathcal{C}(L)$.

Example 12 (The cyclic system). We call cyclic system to the system The system attached to $\Lambda(x)$, a single exterior generator of degree 1 , is called the cyclic system. Clearly, $\mathcal{C}(\Lambda(x))$ consists of an infinite chain of cyclic groups $P_{i} \cong \mathbb{Z} / p^{i+1}$ with limit $P_{\infty} \cong \widehat{\mathbb{Z}}_{p}$. so, this is a system of finite type.

Example 13 (The dihedral system). The system attached to $P[x, y] /\left(x^{2}+x y\right)$ at the prime 2 is called the dihedral system. This system consists of dihedral, generalized quaternion and semidihedral 2-groups. The respective cohomology rings are described in $[12,13]$ (see also [20]). For dihedral groups, $n \geq 1$,

$$
H^{*}\left(D_{2^{n+2}}\right) \cong P[x, y, w] /\left(x^{2}+x y\right)
$$

with $x$ and $y$ of degree 1 and $w$ of degree 2. Clearly, $\rho_{n}: P[x, y] /\left(x^{2}+x y\right) \rightarrow$ $H^{*}\left(D_{2^{n+2}}\right)$ is a 2-equivalence and has codimension 1 in degree 2 . A possible complement $q$ for $\operatorname{Im} \rho_{n}$ in degree 2 is either $w, w+y^{2}, w+x^{2}$ or $w+x^{2}+y^{2}$, and the respective central extensions are either the dihedral group $D_{2^{n+3}}$, for $q=w$, or equivalently for $q=w+x^{2}+y^{2}$, the generalized quaternion group $Q_{2^{n+3}}$, for $q=w+y^{2}$, that gives the cohomology ring

$$
H^{*}\left(Q_{2^{n+3}}\right) \cong P[x, y, v] /\left(x^{2}+x y, y^{3}\right)
$$

where $\operatorname{deg} v=4$, or the semidihedral group $S D_{2^{n+3}}$, for $q=w+x^{2}$, with cohomology ring

$$
H^{*}\left(S D_{2^{n+3}}\right) \cong P[x, y, u, t] /\left(x^{2}+x y, x u, x^{3}, u^{2}+\left(x^{2}+y^{2}\right) t\right)
$$

where $\operatorname{deg} u=3$ and $\operatorname{deg} t=4$. 
Since the given maps

$$
\rho_{n+1}^{\prime}: P[x, y] /\left(x^{2}+x y\right) \rightarrow H^{*}\left(Q_{2^{n+3}}\right)
$$

or

$$
\rho_{n+1}^{\prime \prime}: P[x, y] /\left(x^{2}+x y\right) \rightarrow H^{*}\left(S D_{2^{n+3}}\right)
$$

are 3 -equivalences, the generalized quaternion groups and the semidihedral groups are maximal elements in the system.

Therefore, we have only one infinite chain in the system. The one consisting of the dihedral groups

$$
\cdots \longrightarrow B D_{2^{i+2}} \stackrel{B \pi_{i+1}}{\longrightarrow} B D_{2^{i+1}} \stackrel{B \pi_{i}}{\longrightarrow} \cdots \stackrel{B \pi_{3}}{\longrightarrow} B D_{8}
$$

Notice that the group $D_{2^{n}}$ is a semidirect product $\mathbb{Z} / 2^{n-1} \rtimes \mathbb{Z} / 2$. We see that $\lim D_{2^{n}}=\widehat{\mathbb{Z}}_{p} \rtimes \mathbb{Z} / 2$ with action given by sign change. Let $D_{\infty}$ be the infinite dihedral group $D_{\infty}=\mathbb{Z} \rtimes \mathbb{Z} / 2 \cong \mathbb{Z} / 2 * \mathbb{Z} / 2$. Notice now that the fibration $B \mathbb{Z} \rightarrow$ $B D_{\infty} \rightarrow B \mathbb{Z} / 2$ is homologically nilpotent. This implies that $\lim D_{2^{n}}$ is the $p$ completion of $D_{\infty}$ and that $B D_{\infty}$ is $\mathbb{F}_{p}$-good. Hence

$$
H^{*}\left(\varliminf_{\longleftarrow} D_{2^{n}}\right) \cong H^{*}\left(\left(B D_{\infty}\right)_{p}^{\wedge}\right) \cong H^{*}\left(B D_{\infty}\right) \cong \mathbb{F}_{2}[u, v] / u^{2}+u v \cong \lim _{\longrightarrow} H^{*}\left(D_{2^{n}}\right) .
$$

So, $\mathcal{C}\left(P[x, y] /\left(x^{2}+x y\right)\right)$ is a system of finite type.

We indicate one last example. The system associated to the remaining case of Example 9; that is $L=P[x, y] /\left(x^{2}\right)$ for $p=2$. It is worth mentioning that, although $L$ splits $L \cong \Lambda[x] \otimes P[y]$, the system is not only composed of products of cyclic groups.

Example 14 (The system of $L=P[x, y] /\left(x^{2}\right)$ at the prime 2). For $L=P[x, y] /\left(x^{2}\right)$, $p=2$, the system $\mathcal{C}(L)$ consists of the groups $\mathbb{Z} / 2^{n} \times \mathbb{Z} / 2$, with

$$
H^{*}\left(\mathbb{Z} / 2^{n} \times \mathbb{Z} / 2\right) \cong P[x, y, v] /\left(x^{2}\right) ; \quad \operatorname{deg} v=2
$$

and the non-abelian metacyclic groups $M_{2^{n+2}}, n \geq 2$, defined as the central extension

$$
\mathbb{Z} / 2 \rightarrow M_{2^{n+2}} \rightarrow \mathbb{Z} / 2^{n} \times \mathbb{Z} / 2
$$

classified by the extension class $v+x y \in H^{2}\left(\mathbb{Z} / 2^{n} \times \mathbb{Z} / 2 ; \mathbb{Z} / 2\right)$. Their cohomology, as computed by Rusin [20, Lemma 10] is

$$
H^{*}\left(M_{2^{n+2}}\right) \cong P[x, y, z, t] /\left(x^{2}, x y^{2}, x z, z^{2}\right) ; \quad \operatorname{deg}(z)=3, \operatorname{deg}(t)=4 .
$$

In particular these are maximal elements in the system. Then, the possible infinite chains are chains of products of finite cyclic 2 -groups and $\mathbb{Z} / 2$. The limit of such chains is $\hat{\mathbb{Z}}_{2} \times Z / 2$, and therefore the system is of finite type.

Assume that we have a space $X$ of finite $\mathbb{F}_{p}$-type with $H^{1}(X)=0$ and a map $f: B V \rightarrow X$. If $T_{f}\left(H^{*}(X)\right)$ is of finite type, then we have a 2-approximation $U\left(Q_{2}^{*}\right) \rightarrow U\left(W_{1}^{*}\right) \rightarrow T_{f}\left(H^{*}(X)\right)$. Write $L=U\left(W_{1}^{*}\right) / / U\left(Q_{2}^{*}\right)$, and let $\mathcal{C}(L)$ the associated system of finite $p$-groups. 
Lemma 15. There is a chain of groups in $\mathcal{C}(L), P_{1} \lessdot P_{2} \lessdot \cdots \lessdot P_{i} \cdots$, and a map of towers

such that

$$
g:\left\{E_{s(i)}\right\} \longrightarrow\left\{B P_{i}\right\}
$$

1. For each $i, g_{i}: E_{s(i)} \rightarrow B P_{i}$ induces a commutative diagram

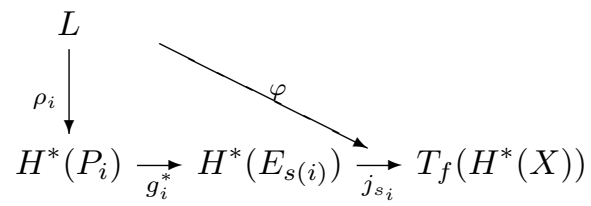

where $\rho_{i}: L \rightarrow H^{*}\left(P_{i}\right)$ is a 2-equivalence.

2. For each $i$, the epimorphism $\pi_{i}: P_{i+1} \rightarrow P_{i}$ satisfies $\pi_{i}^{*}\left(\operatorname{ker}\left(j_{s_{i}} \circ g_{i}^{*}\right)\right)=0$.

Proof. We construct the tower $\left\{B P_{i}\right\}$ and maps $g_{i}: E_{s(i)} \rightarrow B P_{i}$ inductively, starting at $g_{1}: E_{s(1)} \rightarrow B P_{1}$, where $P_{1}$ is the initial object of $\mathcal{C}(L)$ and $g_{1}$ is constructed like in the proof of Theorem 6 .

Assume now that $g_{i}: E_{s(i)} \rightarrow B P_{i}$ has been constructed. Denote $V_{i+1}^{*}=$ $\operatorname{ker}\left(j_{s_{i}} \circ g_{i}^{*}\right) \subset H^{2}\left(B P_{i}\right)$. Since $\rho_{i}$ and $\varphi$ are 2-equivalences, $V_{i+1}^{*} \cap \rho_{i}\left(L^{2}\right)=0$. Then, if we define $P_{i+1}$ by the central extension $V_{i+1} \rightarrow P_{i+1} \stackrel{\pi_{i}}{\longrightarrow} P_{i}$ classified by the inclusion $V_{i+1}^{*} \subset H^{2}\left(B P_{i}\right), P_{i+1}$ belongs to the system $\mathcal{C}(L)$ by the property (A). Furthermore, there is a larger index $s(i+1)$ such that the composition $V_{i+1}^{*} \subset H^{2}\left(B P_{i}\right) \rightarrow H^{2}\left(E_{s(i)}\right) \rightarrow H^{2}\left(E_{s(i+1)}\right)$ is trivial, so that there is a lifting $g_{i+1}: E_{s(i+1)} \rightarrow B P_{i+1}$

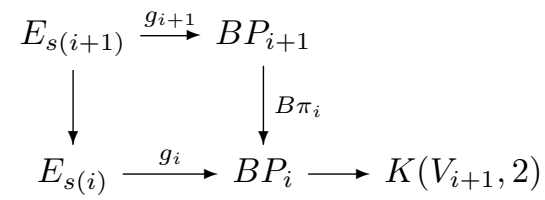

and, by construction, $\pi_{i}^{*}\left(\operatorname{ker}\left(j_{s_{i}} \circ g_{i}^{*}\right)\right)=\pi_{i}^{*}\left(V_{i+1}^{*}\right)=0$.

Theorem 16. Let $X$ be a space of finite $\mathbb{F}_{p}$-type such that $H^{1}\left(X ; \mathbb{F}_{p}\right)=0$ and $f: B V \rightarrow X$ a map. Assume that

$$
U\left(Q_{2}^{*}\right) \stackrel{\psi}{\longrightarrow} U\left(W_{1}^{*}\right) \stackrel{\varphi}{\longrightarrow} T_{f}\left(H^{*}\left(X ; \mathbb{F}_{p}\right)\right)
$$

is a 2-approximation of $T_{f}\left(H^{*}\left(X ; \mathbb{F}_{p}\right)\right)$ and $L=U\left(W_{1}^{*}\right) / / U\left(Q_{2}^{*}\right)$. If the system $\mathcal{C}(L)$ is of finite type, then $f: B V \rightarrow X$ is T-representable.

Proof. According to Lemma 15 we can build a tower $\left\{B P_{i}\right\}$, with $P_{i} \in \mathcal{C}(L)$ and a map $g:\left\{E_{s(i)}\right\} \rightarrow\left\{B P_{i}\right\}$.

Eventually, the tower $\left\{B P_{i}\right\}$ might become constant. This would happen at a stage $g_{i}: E_{s(i)} \rightarrow B P_{i}$ where $V_{i+1}^{*}=\operatorname{ker}\left(j_{s_{i}} \circ g_{i}^{*}\right)=0$ (see the proof of Lemma 15); that is, $j_{s_{i}} \circ g_{i}^{*}: H^{*}\left(P_{i}\right) \rightarrow T_{f}\left(H^{*}(X)\right)$ is a 2-equivalence.

Otherwise we obtain an infinite chain $P_{1} \lessdot P_{2} \lessdot \cdots \lessdot P_{i} \lessdot \cdots$ in $\mathcal{C}(L)$. We have assumed that the system $\mathcal{C}(L)$ is of finite type, hence, if we write $P_{\infty}=\lim _{\longleftarrow} P_{i}$, 
then $H^{*}\left(P_{\infty}\right)=\lim _{\longrightarrow} H^{*}\left(P_{i}\right)$, and this is an $\mathbb{F}_{p^{-}}$-algebra of finite type. Furthermore we obtain an induced map

$$
H^{*}\left(P_{\infty}\right) \rightarrow T_{f}\left(H^{*}(X)\right)
$$

which will be an isomorphism in degree one. Also condition (2) of the tower $\left\{B P_{i}\right\}$ stated in Lemma 15 implies that this is injective, hence a 2-equivalence.

So, in either case, the map of towers $g:\left\{E_{s(i)}\right\} \rightarrow\left\{B P_{i}\right\}$ is a $T$-representation for $f: B V \rightarrow X$.

Remark 17. Notice that our conditions for a finite type system of $p$-groups $\mathcal{C}(L)$ includes already the fact that the pro-finite groups $P_{\infty}$ obtained as limits of infinite chains in $\mathcal{C}(L)$ has finite type mod $p$-cohomology, so the only remaining condition fot showing that $f: B V \rightarrow X$ is finitely $T$-representable is the Tor condition. The counterexamples in the last section will show that this condition cannot be removed.

Remark 18. The cases analyzed in section 4 correspond to the situation in which a system $\mathcal{C}(L)$ consists of a single element. Fix an algebra $L=U\left(W_{1} *\right) / / U\left(Q_{2}^{*}\right)$ and let $P$ be the initial object of the system $\mathcal{C}(L)$; that is, $P$ is the extension $1 \rightarrow Q_{2} \rightarrow P \rightarrow W_{1} \rightarrow 1$ determined by the homomorphism $U\left(Q_{2}^{*}\right) \rightarrow U\left(W_{1}^{*}\right)$. If the induced homomorphism $\rho: L \rightarrow H^{*}(P)$ is a 3 -equivalence, then $P$ is also a maximal element of $\mathcal{C}(L)$, thus $P$ is the only element in $\mathcal{C}(L)$.

Corollary 19. Let $X$ be a space of finite $\mathbb{F}_{p}$-type such that $H^{1}(X)=0$ and $f: B V \rightarrow$ $X$ a map. If there is a 2-equivalence $\Lambda(x) \rightarrow T_{f}\left(H^{*}(X)\right)$, where $\operatorname{deg} x=1$, then $f: B V \rightarrow X$ is T-representable.

This is the special case of $T$-representability that was studied in [1]

Corollary 20. Let $X$ be a space of finite $\mathbb{F}_{p}$-type such that $H^{1}(X)=0$ and $f: B V \rightarrow$ $X$ a map. If there is a 2-equivalence $P[x, y] /\left(x^{2}+x y\right) \rightarrow T_{f}\left(H^{*}(X)\right)$, where $\operatorname{deg} x=$ $\operatorname{deg} y=1$, then $f: B V \rightarrow X$ is T-representable.

The situation encountered in this corollary is significant in the investigation of the homotopy type of the classifying spaces of rank two Kac-Moody groups ([2], $[3])$.

Corollary 21. Let $X$ be a space of finite $\mathbb{F}_{p}$-type such that $H^{1}(X)=0$ and $f: B V \rightarrow$ $X$ a map. If there is a 2-equivalence $P[x, y] /\left(x^{2}\right) \rightarrow T_{f}\left(H^{*}(X)\right)$, where $\operatorname{deg} x=$ $\operatorname{deg} y=1$, then $f: B V \rightarrow X$ is T-representable.

Remark 22. As a final remark, let us note that all our above calculations assume only very little knowledge of the structure of $T_{f} H^{*} X$. This has the advantage that this structure can be recognized very easily. However, in many applications a richer structure can be successfully applied. These include the algebra structure in higher degrees, and also Steenrod operations in low degrees. A very important tool in many cases might be the Bockstein spectral sequence. This makes sense, since $T_{f} H^{*} X$ inherits a Bockstein spectral sequence from the isomorphism 
$T_{f} H^{*} X \cong \lim _{1} H^{*}\left(E_{s}\right)$. If we have information about the higher Bockstein operations in $T_{f} \overrightarrow{H^{*}} X$ we can obtain finite $T$-representability in cases where the fundamental group of the mapping space $\operatorname{Map}\left(B V, X_{p}^{\wedge}\right)_{f}$ is a finite abelian $p$-group, not necessarily elementary abelian.

\section{A Counterexample}

In this section we show a family of examples in which a particular component of a mapping space with source $B \mathbb{Z} / p$ and target a 1-connected $p$-complete space of finite $\mathbb{F}_{p}$-type is not $p$-good, hence $T$ does not compute its cohomology.

The idea is simple. Choose a space $X$ which is 1-connected and of finite $\mathbb{F}_{p^{-}}$ type, a non trivial map $f: B \mathbb{Z} / p \rightarrow X$, and a finite complex $B$ which is $p$-bad. The space $W=X \wedge B_{+}$will also be 1-connected and of finite $\mathbb{F}_{p}$-type, however for the composition $w: B \mathbb{Z} / p \rightarrow X \rightarrow W$, the mapping space $\operatorname{Map}\left(B \mathbb{Z} / p, W_{p}^{\wedge}\right)_{w}$ is likely to be $p$-bad. We will work out the particular case $X=\mathbb{C} P^{\infty}, f: B \mathbb{Z} / p \rightarrow \mathbb{C} P^{\infty}$ a non trivial map, and $B=S^{1} \vee S^{m}$.

Define $Y=\mathbb{C} P^{\infty} \times\left(S^{1} \vee S^{m}\right)$, and $W$ as the homotopy cofibre of $S^{1} \vee S^{m} \rightarrow Y$; that is $W=\mathbb{C} P^{\infty} \wedge\left(S^{1} \vee S^{m}\right)_{+}$. We can easily obtain the mod $p$ cohomology algebras of these spaces. We choose an odd prime $p$, although similar considerations can be made for $p=2$ :

$$
\begin{aligned}
& H^{*}(Y) \cong P[e] \otimes \Lambda[a, b] /(a b), \quad \operatorname{deg} e=2, \operatorname{deg} a=1, \operatorname{deg} b=m \\
& H^{*}(W) \cong P[e] \otimes \Lambda[x, y] /(x y), \quad \operatorname{deg} x=3, \operatorname{deg} y=m+2 .
\end{aligned}
$$

Moreover, the map $g: Y \rightarrow W$, from $Y$ to the homotopy cofibre, induces in cohomology $g^{*}(e)=e, g^{*}(x)=e a$, and $g^{*}(y)=e b$. It follows that $\mathcal{P}^{1} x=x e^{p-1}$ and $\mathcal{P}^{1} y=y e^{p-1}$. Notice that $W$ is 1 -connected of finite $\mathbb{F}_{p^{-} \text {-type. }}$

The map $B \mathbb{Z} / p \times \mathbb{C} P^{\infty} \rightarrow \mathbb{C} P^{\infty}$ induced by the multiplication $\mathbb{Z} / p \times S^{1} \rightarrow S^{1}$, extends to a map

$$
\mu: B \mathbb{Z} / p \times \mathbb{C} P^{\infty} \times\left(S^{1} \vee S^{m}\right) \longrightarrow \mathbb{C} P^{\infty} \times\left(S^{1} \vee S^{m}\right) \longrightarrow \mathbb{C} P^{\infty} \wedge\left(S^{1} \vee S^{m}\right)_{+} ;
$$

that is, $\mu: B \mathbb{Z} / p \times Y \rightarrow W$. If we write $H^{*}(B \mathbb{Z} / p)=\Lambda[u] \otimes P[v]$, the map in cohomology induced by $\mu$ is $\mu^{*}: H^{*}(W) \rightarrow H^{*}(B \mathbb{Z} / p) \otimes H^{*}(Y)$, determined by $\mu^{*}(e)=v \otimes 1+1 \otimes e, \mu^{*}(x)=1 \otimes a e$, and $\mu^{*}(y)=1 \otimes b e$.

Lemma 23. Let $w: B \mathbb{Z} / p \rightarrow W$ the restriction of $\mu$ to $B \mathbb{Z} / p$ at the base point of $Y$. The adjoint of $\mu^{*}$ is an isomorphism $\tilde{\mu}^{*}: T_{w}\left(H^{*}(W)\right) \rightarrow H^{*}(Y)$.

Proof. We sketch the proof. It follows the methods of $[1, \S 3] . H^{*}(W)$ fits in an exact sequence of unstable $H^{*}(W)-\mathcal{U}$-modules that can also be seen as $P[e]-\mathcal{U}$-modules

$$
0 \longrightarrow x P[e] \otimes y P[e] \longrightarrow H^{*}(W) \longrightarrow P[e] \longrightarrow 0 .
$$

Since $x P[e] \cong y P[e] \cong \Sigma e P[e]$ as unstable $P[e]-\mathcal{U}$-modules, where $\Sigma$ denotes the suspension functor, we obtain $T_{w}(x P[e]) \cong T_{w}(y P[e]) \cong \Sigma T_{w}(e P[e]) \cong$ 
$\Sigma P[e]=z P[e]$, with trivial Steenrod algebra action on $z$. Hence, there is an exact sequence

$$
0 \longrightarrow a P[e] \otimes b P[e] \longrightarrow T_{w}\left(H^{*}(W)\right) \longrightarrow P[e] \longrightarrow 0
$$

and the induced homomorphism $\varepsilon: H^{*}(W) \rightarrow T_{w}\left(H^{*}(W)\right)$ maps $\varepsilon(e)=e, \varepsilon(x)=$ $a e$, and $\varepsilon(y)=b e$. Since the composition $H^{*}(W) \stackrel{\varepsilon}{\rightarrow} T_{w}\left(H^{*}(W)\right) \stackrel{\tilde{\mu}^{*}}{\longrightarrow} H^{*}(Y)$ should coincide with $g^{*}$, it turns out that $\tilde{\mu}^{*}: T_{w}\left(H^{*}(W)\right) \rightarrow H^{*}(Y)$ is an isomorphism.

Corollary 24. $\mu$ induces a homotopy equivalence $Y_{p}^{\wedge} \stackrel{\simeq}{\longrightarrow} \operatorname{Map}\left(B \mathbb{Z} / p, W_{p}^{\wedge}\right)_{w}$.

Proof. It follows directly from Lemma 23 above and [15, 3.3.2].

According to [6], $S^{1} \vee S^{m}$ is $\mathbb{F}_{p}$-bad, hence, so is $Y=\mathbb{C} P^{\infty} \times\left(S^{1} \vee S^{m}\right)$. More precisely, Bousfield shows that, for $m \geq 2, H_{2 m}\left(\left(S^{1} \vee S^{m}\right)_{p}^{\wedge}\right)$ is uncountable, and this implies that $H_{2 m}\left(Y_{p}^{\wedge}\right)$ is uncountable, as well. Also, in the case $m=1$, either $H_{2}\left(\left(S^{1} \vee S^{1}\right)_{p}^{\wedge}\right)$ or $H_{3}\left(\left(S^{1} \vee S^{1}\right)_{p}^{\wedge}\right)$ is uncountable, and the same will be true for $H_{*}\left(Y_{p}^{\wedge}\right)$. This means that, definitely, $T_{w}\left(H^{*}(W)\right)$ is not isomorphic to $H^{*}\left(\operatorname{Map}\left(B \mathbb{Z} / p, W_{p}^{\wedge}\right)_{w}\right)$.

For $m=1, T_{w}\left(H^{*}(W)\right)$ is not $T$-representable. $\Lambda[a, b] /(a b) \rightarrow T_{w}\left(H^{*}(W)\right)$ is a 2-approximation, but the possible towers of groups $\left\{G_{s}\right\}$ associated to this 2-approximation would give $G_{\infty}=\lim _{G_{s}}=(\mathbb{Z} * \mathbb{Z})_{p}^{\wedge}$, the $p$-profinite completion of $\mathbb{Z} * \mathbb{Z}$, which is the fundamental group of $\left(S^{1} \vee S^{1}\right)_{p}^{\wedge}$, but in this case $\stackrel{\lim }{\longrightarrow} H^{*}\left(G_{s}\right) \neq$ $H^{*}\left(G_{\infty}\right)$.

For $m \geq 2, T_{w}\left(H^{*}(W)\right)$ is $T$-representable according to Example 12, but it is not finitely $T$-representable because $\operatorname{Tor}_{\Lambda(a)}^{*, *}\left(T_{w}\left(H^{*}(W)\right), \mathbb{F}_{p}\right)$ is not finite dimensional in each total degree. We can decompose

$$
T_{w}\left(H^{*}(W)\right) \cong P[e] \otimes \Lambda[a, b] /(a b) \cong(P[e] \otimes \Lambda[a]) \oplus b P[e]
$$

as $\Lambda[a]$-modules, and then

$$
\begin{aligned}
& \operatorname{Tor}_{\Lambda(a)}^{*, *}\left(T_{w}\left(H^{*}(W), \mathbb{F}_{p}\right) \cong \operatorname{Tor}_{\Lambda(a)}^{*, *}\left((P[e] \otimes \Lambda[a]) \oplus b P[e], \mathbb{F}_{p}\right)\right. \\
& \quad \cong \operatorname{Tor}_{\Lambda(a)}^{*, *}\left(P[e] \otimes \Lambda[a], \mathbb{F}_{p}\right) \oplus \operatorname{Tor}_{\Lambda(a)}^{*, *}\left(b P[e], \mathbb{F}_{p}\right) \cong P[e] \oplus\left(\Gamma\left[\gamma_{0}\right] \otimes b P[e]\right)
\end{aligned}
$$

where $\Gamma\left[\gamma_{0}\right]$ is an algebra of divided powers on a generator of total degree zero, thus making $\operatorname{Tor}_{\Lambda(a)}^{*, *}\left(T_{w}\left(H^{*}(W)\right), \mathbb{F}_{p}\right)$ infinite dimensional in total degree zero.

This last example shows also that the condition on Tor cannot be removed from the hypothesis of the main theorem.

\section{References}

[1] J. Aguadé, C. Broto, D. Notbohm, Homotopy classification of spaces with interesting cohomology and a conjecture of Cooke. Part I. Topology 33 (1994), 455-492.

[2] J. Aguadé, C. Broto, N. Kitchloo, L. Saumell, Homotopy type of classifying spaces of rank two Kac-Moody groups. (In preparation.) 
[3] J. Aguadé, C. Broto, N. Kitchloo, L. Saumell, Cohomology of classifying spaces of central quotients of rank two Kac-Moody groups. Preprint.

[4] D. J. Benson, J. F. Carlson, The cohomology of extraspecial groups, Bull. London Math. Soc. 24 (1992), 209-235. Erratum to: "The cohomology of extraspecial groups" , Bull. London Math. Soc. 25 (1993), 498.

[5] A. K. Bousfield, On the homology spectral sequence of a cosimplicial space. Amer. J. Math. 109 (1987), 361-394.

[6] A. K. Bousfield, On the p-adic completions of nonnilpotent spaces. Trans. Amer. Math. Soc. 331 (1992), 335-359.

[7] A. K. Bousfield, D. M. Kan, Homotopy limits, completions and localizations. Lecture Notes in Math., 304. Springer-Verlag, Berlin-New York, 1972.

[8] C. Broto, R. Levi, Loop structures on homotopy fibres of self maps of spheres. Amer. J. Math. 122 (2000), 547-580.

[9] T. Diethelm, The mod p cohomology rings of the nonabelian split metacyclic p-groups. Arch. Math. 44 (1985), 29-38.

[10] E. Dror Farjoun, J. Smith, A geometric interpretation of Lannes' functor T. Astérisque, 191 (1990), 87-95.

[11] W. G. Dwyer, Strong convergence of the Eilenberg-Moore spectral sequence. Topology 13 (1974), 255-265.

[12] L. Evens, S. Priddy, The cohomology of the semidihedral group. Conference on algebraic topology in honor of Peter Hilton (Saint John's, Nfld., 1983), 61-72, Contemp. Math., 37, Amer. Math. Soc., Providence, RI, 1985.

[13] Z. Fiedorowicz, S. Priddy, Homology of classical groups over finite fields and their associated infinite loop spaces. Lecture Notes in Math., 674. Springer, Berlin, 1978.

[14] C. U. Jensen, Les foncteurs dérivés de lim et leurs applications en théorie des mod-

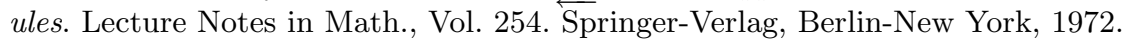

[15] J. Lannes, Sur les espaces fonctionnels dont la source est le classifiant d'un p-groupe abélien élémentaire. Publ. Math. I.H.E.S., 75 (1992), 135-244.

[16] I. J. Leary, The mod-p cohomology rings of some p-groups, Math. Proc. Cambridge Philos. Soc. 112 (1992), 63-75.

[17] F. Morel, Quelques remarques sur la cohomologie modulo p continue des pro-p-espaces et les résultats de J. Lannes concernant les espaces fonctionnels hom $(B V, X)$. Ann. Sci. École Norm. Sup., 26 (1993), 309-360.

[18] F. Morel, Ensembles profinis simpliciaux et interprétation géométrique du foncteur T. Bull. Soc. math. France, 124 (1996), 347-373.

[19] D. Quillen, The mod 2 cohomology rings of extra-special 2-groups and the spinor groups, Math. Ann. 194 (1971) 197-212.

[20] D. J. Rusin, The mod 2 cohomology of metacyclic 2-groups, J. Pure Appl. Algebra 44 (1987), 315-327.

Dep. de Matemàtiques, Universitat Autònoma de Barcelona, E-08193 Bellaterra, Spain

E-mail address: aguade@mat.uab.es broto@mat.uab.es laia@mat.uab.es 